\title{
Visual discrimination of texture
}

\author{
RANDI C. MARTIN \\ The Johns Hopkins University, Baltimore, Maryland 21218 \\ and \\ JAMES R. POMERANTZ \\ State University of New York at Buffalo, Buffalo, New York 14226
}

\begin{abstract}
Julesz (1975) proposed a theory of texture discrimination, based on an order statistics principle, which states that no two textures can be perceptually discriminated if they have identical second-order statistics. The experiments reported here demonstrate that this principle is not adequate to predict visual texture discriminability. Both letter and dot micropatterns were used to create texture pairs that either differed or were identical with respect to secondorder statistics. The subject's task was to decide which quadrant of an array contained a disparate texture. In Experiments 1, 2, and 3, when controlling for spatial overlap, texture pairs having identical second-order statistics were discriminated more quickly than similar texture pairs having different second-order statistics, in contradiction to the principle. Although a significant effect in the direction predicted by the order statistics principle was found in Experiment 4 for texture pairs created from the dot micropatterns, other factors, such as spatial overlap, border differences, and goodness of pattern, must also be considered in predicting texture discriminability.
\end{abstract}

Texture discrimination is an important process in vision that provides cues for depth perception, figureground segregation, and form recognition (Gibson, 1950). Despite its importance, there has been little work to date on identifying the stimulus properties that make textures distinguishable. One exception has been the work of Julesz $(1962,1973,1975)$, who has devised a formal measure of textural properties based on order statistics. On the basis of his findings with this measure, Julesz has proposed a principle which states that no two textures can be perceptually discriminated if they have identical second-order statistics. Julesz has created several demonstrations that provide compelling confirmation of his hypothesis. These demonstrations have been published, but no formal experiment relating to this principle has been reported. The experiments we report below test the principle experimentally, indicate that this principle is not adequate, and suggest that other principles need be advanced to account for texture discrimination.

\section{Order Statistics}

First-order statistics (OS), which correspond phenomenally to the average brightness of a texture, are measured by the probability that a point thrown randomly on a texture would land on a

This research was supported in part by a grant from the National Science Foundation to Howard Egeth and to James Pomerantz (BNS 76 01227). Requests for reprints should be sent to James Pomerantz, Department of Psychology, SUNY at Buffalo, 4230 Ridge Lea Road, Buffalo, New York 14226. black dot. Imagine two white patches of equal area sprinkled with black dots of equal size to create a texture. If one patch contained more black dots than the other, the two textures would differ in the first OS. Second OS, which correspond phenomenally to the granularity or uniformity of a texture, are measured by determining the probability that pins (or dipoles) of all possible lengths and orientations thrown on a texture would land with both ends on black dots. Consider two textures that have the same density of black dots, If, on the first texture, no two dots are allowed to fall closer than some predetermined distance while, on the second, there is no such constraint, the two textures would differ in their second but not in their first OS. ${ }^{1}$ The average brightness of the two textures would be the same, but the unconstrained texture would appear more "clumpy." Third OS are not easy to describe, but are measured in a manner similar to second OS; they refer to the probability that randomly thrown triangles of fixed size, shape, and orientation would land with all three vertices on black. Two textures that have the same second OS may differ in third OS.

According to Julesz' principle, textures that differ in their first OS or second OS should be readily discriminable. Textures that agree in their first OS and second OS, even if they differ in third OS, should not be perceptually discriminable except under close scrutiny. Julesz (1975) has presented some weak counterexamples to this principle, such as textures which agree in second OS but which are 
barely discriminable. We present below counterexamples of textures that agree in second OS but that are not only easily discriminable, but are even more discriminable than textures that differ in second OS.

Consider textures made up of small geometric figures (called micropatterns), such as the ones shown in Figure 1a. Any individual micropattern

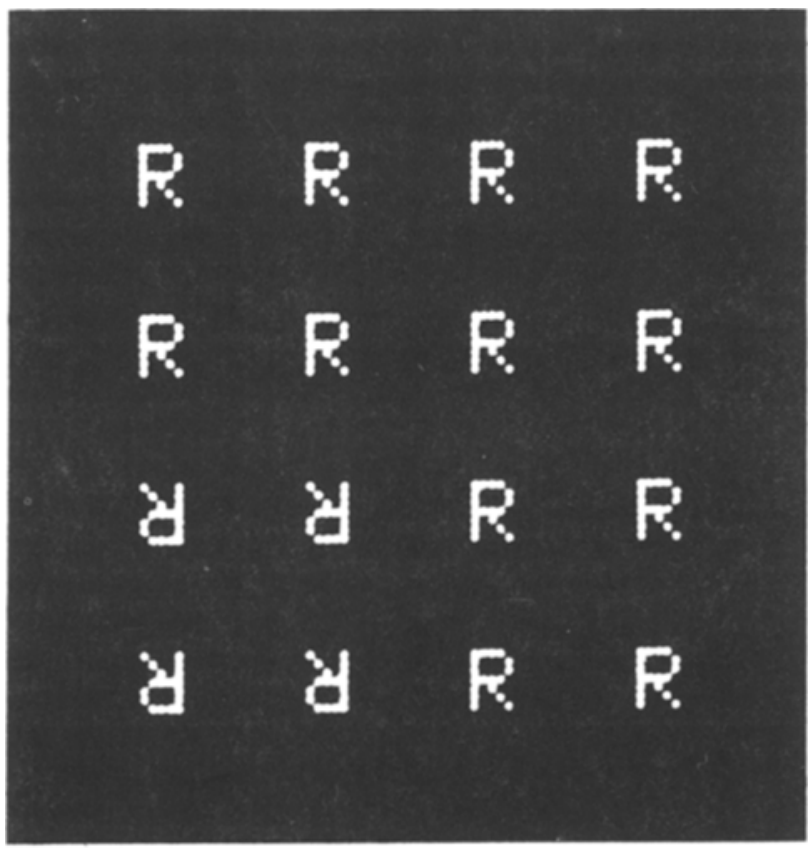

a.

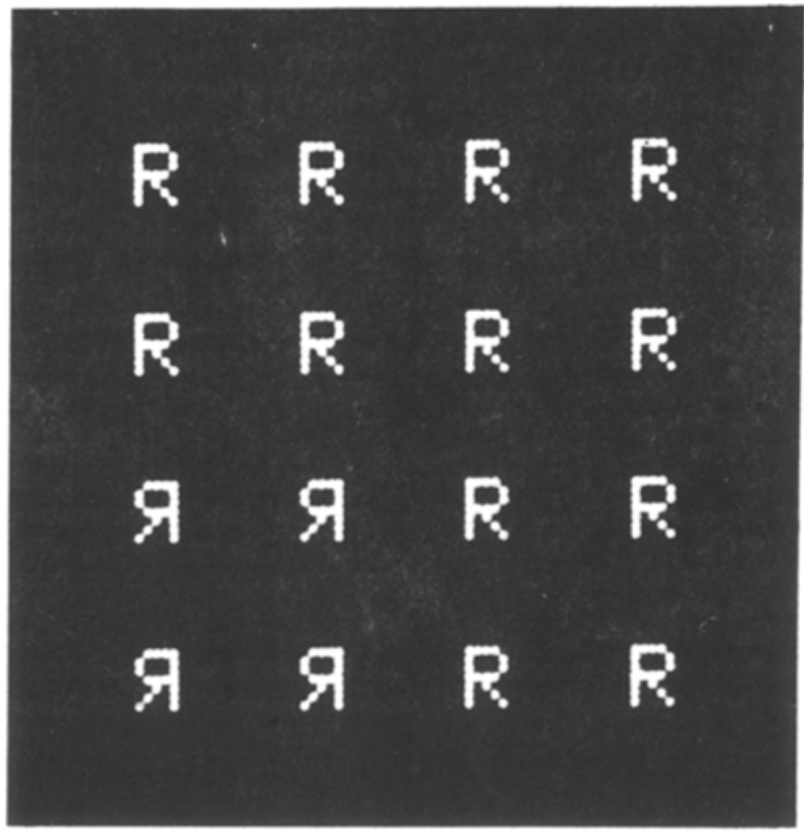

b.

Figure 1. (a) The $R$ and inverted $R$ micropatterns have the same second $O S$. (b) The $R$ and mirror-image $R$ micropatterns have different second $O S$. and its $180^{\circ}$ rotation must share the same second OS, since any pin landing on the micropattern with both ends on white (in this example, the background is black and the micropattern white) could be matched by a pin of identical length and orientation landing with both ends on white on the $180^{\circ}$ rotation. Similarly, textures consisting of such micropatterns sprinkled at regularly spaced positions must also agree in their second OS (Gilbert \& Shepp, Note 1). One demonstration Julesz has created to support his principle consists of one texture made up of upright Us, another of inverted Us, and a third of sideways Us (or Cs). The upright- and inverted-U textures agree in second OS (but not in third OS), while the upright and sideways textures differ in second OS. In accord with the principle, the first pair of textures is quite difficult to discriminate while the second is quite easy.

Although this example supports the order statistics principle, another possible explanation of the difficulty in discriminating the upright Us from the $180^{\circ}$-rotation Us relative to the difficulty in discriminating the upright Us from the sideways Us is that the $U$ and its $180^{\circ}$ rotation overlap in a templatematching sense to a greater extent than do the $U$ and sideways $U$. To test this possibility, we created textures generated from other letter micropatterns that either agreed or disagreed in second OS, but where the degree of overlap would not be confounded with differences in order statistics.

\section{EXPERIMENT 1}

In this experiment, we created textures that used as a micropattern the letter $\mathbf{R}$ in three different orientations : upright (normal), $180^{\circ}$ rotation in the plane, and mirror reversal. The upright and rotated $R$ textures agree in the first and second OS, while the upright and reversed textures agree in first but differ in second OS. Unlike the $U$ micropatterns, the micropatterns differing in second $O S(R$ and mirror-image $\mathbf{R}$ ) would overlap to the same extent as the micropatterns having identical second OS $(R$ and $180^{\circ}$ rotation $R$ ) when positioned to maximize degree of overlap (see Figure 2). That the upright and rotated $\mathbf{R}$ textures would agree in second OS can be seen by noting that every pin landing with both ends on the diagonal of the $R$ could be matched with a pin landing on the diagonal of the $180^{\circ}$ rotaton, since the diagonals of the $R$ and $180^{\circ}$ rotation are of the same orientation. However, for the mirror-image $R$, these pins could not be matched since the diagonal of the mirror image $R$ is of a different orientation than diagonal of the $\mathbf{R}$. Thus, the upright and mirror-image $R s$ must differ in second OS.

The order statistics principle predicts that the 
upright- and rotated-R textures should be difficult to discriminate, while the upright- and reversed-R textures should be easy to discriminate. Based on degree of overlap, there should be no difference in discriminability between the texture pairs.

\section{Method}

Subjects. Eleven college students with normal vision participated in this experiment for course credit.

Stimuli. Arrays like those shown in Figures 1a and $1 \mathrm{~b}$ were used. The arrays contained 16 micropatterns arranged in a square matrix. Twelve of the micropatterns were identical, while the remaining four, located in one quadrant of the matrix, were different from these 12 . The three quadrants containing identical micropatterns will be referred to as the surround, and the remaining quadrant, the odd, or disparate, quadrant. The arrays contained upright-R micropatterns paired with either the mirror-image $\mathrm{Rs}$ or the $180^{\circ}$ rotations. The upright $\mathrm{Rs}$ filled the surround on half the arrays and filled the odd quadrant on the other half. The arrays that contained the upright $\mathrm{Rs}$ in the odd quadrant will be referred to as reversals of the arrays which contained the same two textures, but with the upright Rs filling the surround. For each surround-odd quadrant texture pair, there were four different arrays, one for each possible disparate quadrant. Thus, there were 16 different stimulus arrays in all.

The textured pairs were presented on the screen of a computercontrolled cathode ray tube. The arrays subtended a visual angle of $8^{\circ}$, which is well within the size of the examples presented in print by Julesz $(1973,1975)$ when his figures are held at normal viewing distance. ${ }^{2}$ The arrangement of micropatterns into regular rows and columns was selected to parallel Julesz' arrays with the $\mathrm{U}$ micropatterns discussed above. The individual micropatterns subtended approximately $55^{\prime}$ visual angle, while the spaces between them subtended $1^{\circ} 25^{\prime}$.

Procedure. Subjects were required to determine as quickly as possible which quadrant contained the disparate array. They indicated their responses by pushing the top button of two vertically arranged buttons if the disparate quadrant was in the top half of the array and the lower button if it was in the lower half of the array.

Stimuli were presented in trial blocks containing all 16 arrays. A different random order was used for each block. The first block was considered practice. Ten experimental blocks followed the practice block.

Each trial was preceded by a fixation dot on an otherwise blank screen. The array remained on the screen until a response was made.
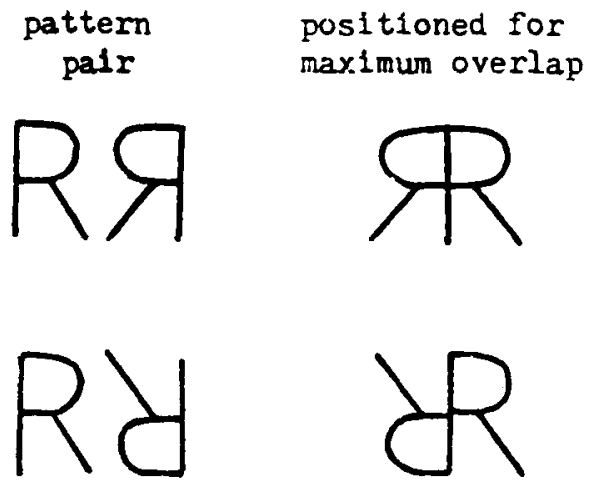

Figure 2. The $R$ and mirror-image $R$ and the $R$ and $180^{\circ}$-rotation $R$ overlap to the same extent when positioned to maximize overlap.
Table 1

Mean Reaction Times (in Milliseconds) for Textures Consisting of $R$ and L Micropatterns (16 Pattern Arrays)

\begin{tabular}{|c|c|c|c|c|c|c|c|}
\hline \multicolumn{8}{|c|}{ Order Statistics } \\
\hline \multicolumn{4}{|c|}{ Second Order Same } & \multicolumn{4}{|c|}{ Second Order Different } \\
\hline $\begin{array}{l}\text { Pat- } \\
\text { tern* }\end{array}$ & RT & $\begin{array}{l}\text { Error } \\
\text { Rate } \dagger\end{array}$ & $\begin{array}{c}\text { Mean } \\
\text { RT }\end{array}$ & $\begin{array}{l}\text { Pat- } \\
\text { tern* }\end{array}$ & RT & $\begin{array}{l}\text { Error } \\
\text { Rate } \dagger\end{array}$ & $\begin{array}{c}\text { Mean } \\
\text { RT }\end{array}$ \\
\hline $\begin{array}{ll}\mathrm{R} & \mathrm{H} \\
\mathrm{C} & \mathrm{R}\end{array}$ & $\begin{array}{l}927 \\
969\end{array}$ & $\begin{array}{l}1.4 \\
2.4\end{array}$ & 948 & $\begin{array}{ll}\mathrm{R} & \mathrm{G} \\
\text { Я } & \mathrm{R}\end{array}$ & $\begin{array}{r}981 \\
1,075\end{array}$ & $\begin{array}{l}3.4 \\
5.2\end{array}$ & 1,028 \\
\hline${ }_{\mathrm{T}}^{\mathrm{L}} \mathrm{T}$ & $\begin{array}{r}972 \\
1,008\end{array}$ & $\begin{array}{l}1.1 \\
1.6\end{array}$ & 990 & $\frac{L}{I} \frac{I}{L}$ & $\begin{array}{r}973 \\
1,095\end{array}$ & $\begin{array}{l}1.4 \\
2.3\end{array}$ & 1,034 \\
\hline
\end{tabular}

*Surround-odd.

IIn percentages.

\section{Results}

Mean reaction times (RTs) are shown in Table 1. A two-way analysis of variance of texture pairs (second-order statistics same vs. second-order statistics different) by reversals (upright-R surround or upright- $R$ disparate) showed a significant main effect for texture pair $[F(1,10)=5.7, p<.05]$ and for reversals $[F(1,10)=15.7, p<.01]$. The interaction was not significant. The results for texture pairs indicated that the pairs with second-order statistics the same were discriminated more quickly (mean RT $=948$ ) than were the pairs with secondorder statistics different (mean RT $=1,028$ ). This result was thus significant in the direction opposite that predicted by the order statistics principle.

The main effect for reversals indicated that the arrays with the upright $R s$ in the surround were discriminated more quickly (mean RT $=954$ ) than were those with the upright Rs filling the disparate quadrant (mean RT $=1,022$ ). This result replicates earlier findings (Frith, 1974; Reicher, Snyder, \& Richards, 1976), which have indicated that an unfamiliar pattern (mirror-image $R$ or $180^{\circ}$ rotaton) is easier to locate in a field of familiar patterns (upright $\mathbf{R}$ ) than is a familiar pattern in an unfamiliar field. While this finding is not directly in contrast to any prediction made by the order statistics principle, it implies that additional principles must be postulated in order to predict texture discriminability, since the statistical differences between two textures would remain the same regardless of which texture formed the background.

\section{EXPERIMENT 2}

To verify that our results were not peculiar to the $\mathbf{R}$ micropatterns, we performed a second, otherwise identical, experiment using textures made from Ls in their upright, rotated, and reverse orientations (see Figures $3 a$ and $3 b$ ). The $L$ and $180^{\circ}$-rotation $L$ would have the same second OS, while the $\mathrm{L}$ and the mirror-image $\mathrm{L}$ would differ in second OS. 


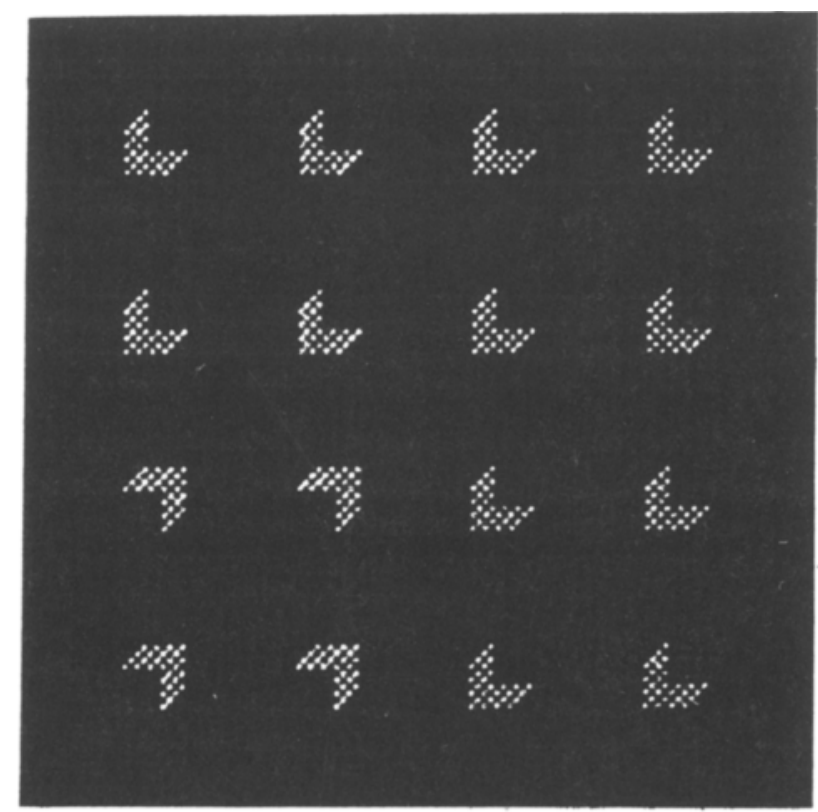

a.

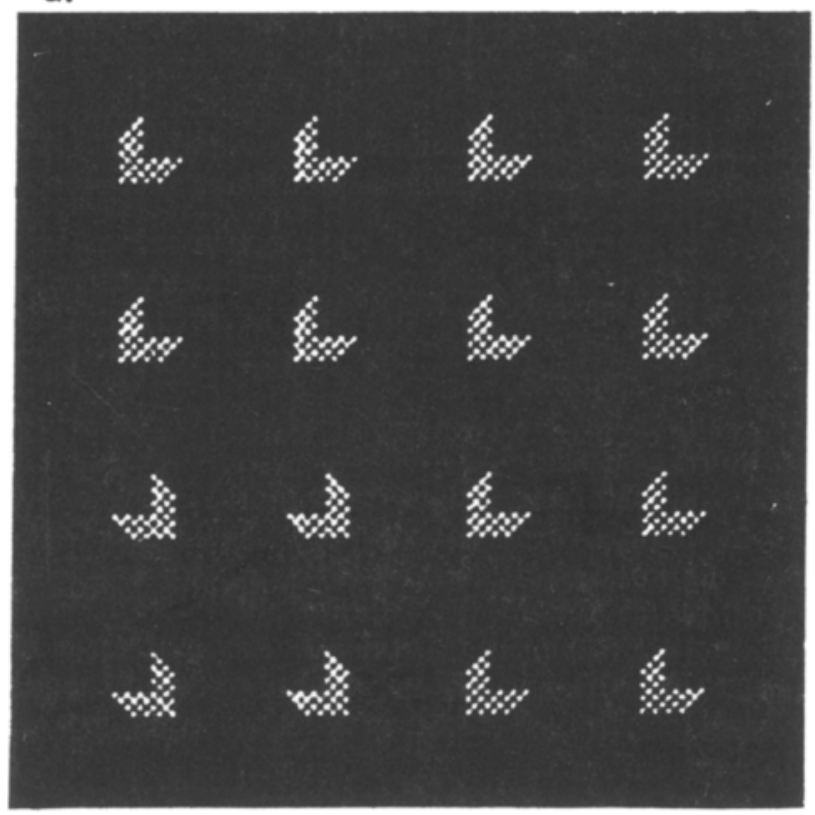

b.

Figure 3. (a) The $L$ and inverted-L micropatterns bave the same second $O S$. (b) The $L$ and mirror-image $L$ have different second $O S$.

Again, the micropatterns differing in second OS would overlap to the same extent as the micropatterns having the same second OS. That the $L$ and $180^{\circ}$ rotation $\mathrm{L}$ would have the same second OS can be seen by noting that all pins landing with one end on each of the lines making up the $L$ would be of negative slope, as would all pins landing with one end on each side of the $180^{\circ}$ rotaton. However, for the mirror-image $\mathrm{L}$, all pins landing with one end on each side would be of positive slope. Thus, the pins landing on the $\mathrm{L}$ could be matched in length and orientation with pins landing on the $180^{\circ}$ rotation, but not with pins landing on the mirror image.

\section{Method}

Subjects. The same 11 subjects from Experiment 1 participated in this experimient for course credit.

Stimuli. The arrays were constructed in the same manner as those used in Experiment 1, with the exception that Ls were used rather than Rs; upright $\mathrm{Ls}$ were paired with either Ls totated $180^{\circ}$ or with mirror-image Ls.

Procedure. The procedure was identical to that used in Experiment 1.

\section{Results}

Mean RTs are again shown in Table 1. A two-way analysis of variance of texture pairs (second-order statistics same vs. second-order statistics different) by reversals (upright $L$ in surround or in disparate quadrant) showed a significant main effect for reversals only $[F(1,10)=6.18, p<.05]$. The interaction was not significant. The main effect for reversals indicated that those pairs where the upright Ls filled the surround were discriminated more quickly (mean RT $=972$ ) than those where the upright Ls filled the disparate quadrant (mean RT = 1,052).

Although the main effect for texture pairs was not significant, the results were in the direction opposite that predicted by the order statistics principle. Eight of 11 subjects discriminated texture pairs that agreed in second OS faster than texture pairs differing in second OS. While this result is not as striking as that found in the first experiment, it does not support the order statistics principle, since, according to this principle, texture pairs agreeing in second OS should have been much more difficult to discriminate, and so a strong result in the opposite direction should have appeared.

\section{Discussion}

The order statistics principle states that two textures that agree in second-order statistics cannot be perceptually discriminated. The approach taken in these experiments was to show that, when controlling for degree of overlap, textures which agree in second OS would take no longer to discriminate than would textures with different second OS. In both experiments, it was found that textures that agreed in second OS were actually easier to discriminate than those having different second $O S$.

In view of the long RTs obtained in these studies (averaging over $1,000 \mathrm{msec}$ ), it might be argued that none of the discriminations we tested were perceptual, but rather all of them required scrutiny (or focused attention). However, such lengthy RTs are not uncommon, even for the simplest texture discriminations 
(cf. Olson \& Attneave, 1970). For one specific example, Pomerantz, Sager, and Stoever (1977) obtains RTs in the $908-993 \mathrm{msec}$ range for discriminating texture pairs that differed in first as well as second OS (p. 428, Figures 14-17). The length of the RTs in these texture localization tasks appears to stem in part from response components of task. ${ }^{3}$

\section{EXPERIMENT 3}

To generalize our results further, we tested textures similar to others constructed by Julesz that use dot configurations as micropatterns rather than familiar letter-like forms. The method for constructing such textures having identical second OS is shown in Figure 4 and has been described in detail elsewhere (Julesz, 1975; Gilbert \& Shepp, Note 1). One begins with three dots (X, Y, and Z), which form a triangle. The midpoint (M) of a line connecting two of the dots ( $\mathrm{X}$ and $\mathrm{Y}$ ) is located, and a line connecting this midpoint with the third $\operatorname{dot}(\mathrm{Z})$ is drawn. Then a perpendicuiar to this line through $M$ is found. Two dots ( $P$ and $Q$ ) are located on this perpendicular such that the length of $\overline{\mathrm{MP}}$ is equal to the length of $\overline{\mathrm{MQ}}$. Considering the pattern formed by $X$, $Y, Z$, and $P$ and the pattern formed by $X, Y, Z$, and $Q$ as two different micropatterns, it can be shown that these micropatterns would have all interpoint distances equal. First, $\mathrm{X}, \mathrm{Y}$, and $\mathrm{Z}$ are common to both patterns; thus, all interpoint distances among these three points would be the same. Secondly, from

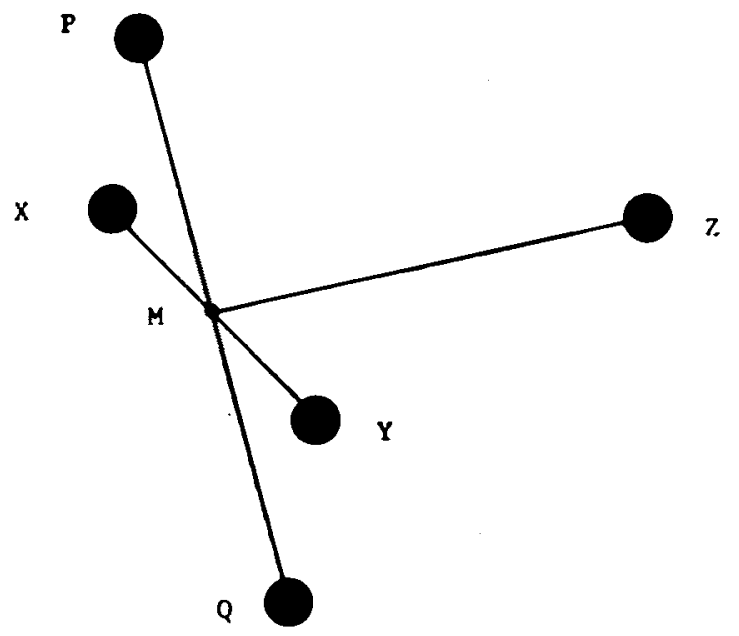

Figure 4. This figure shows the method of constructing dot micropatterns having identical second-order statistics. $M$ is the midpoint of $\overline{X Y}$. Line $\overline{P Q}$ is perpendicular to line $\overline{M Z}$. $P$ and $Q$ are equidistant from $M$. From the geometry of the figure, it can be shown that a micropattern consisting of points $X, Y, Z$, and $P$ would have all interpoint distances equal to those of a micropattern consisting of points $X, Y, Z$, and $Q$.
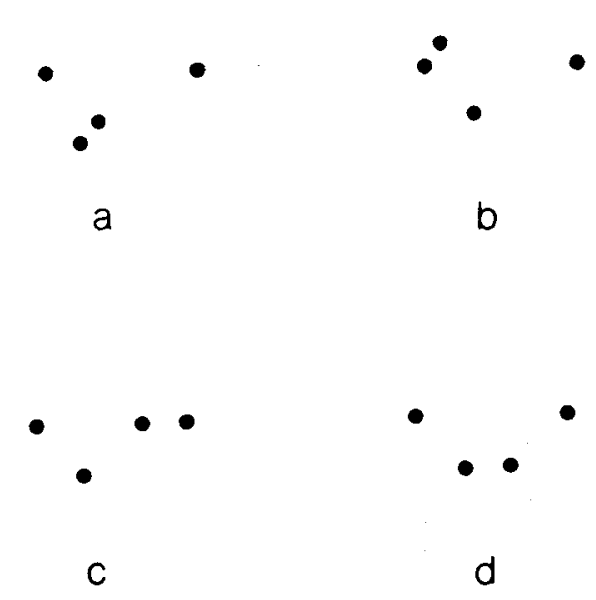

Figure 5. Dot micropatterns having three dots in common. Micropattern pair a and $b$ and micropattern pair $c$ and $d$ have identical second-order statistics. The remainins pairs (a and $c$, $a$ and $d, b$ and $c$, and $b$ and d) have different second-order statistics.

the geometry of the figure, it is evident that $\overline{\mathrm{XP}}=$ $\overline{\mathrm{YQ}}, \overline{\mathrm{PZ}}=\overline{\mathrm{QZ}}$, and $\overline{\mathrm{YP}}=\overline{\mathrm{XQ}}$. Hence, a pin of a certain length would have an equal probability of landing with both ends on black for both micropatterns. However, orientations of pins would not all match. For example, if the micropatterns were oriented as shown in Figure 4, the orientation of $\overline{\mathrm{ZP}}$ would differ from the orientation of $\overline{\mathrm{ZQ}}$. One method of equalizing orientations (and hence second OS) is to randomly rotate the micropatterns making up a texture (see Figure 6).

Julesz has presented texture pairs created from micropatterns of shape similar to those shown in Figure 4, and as predicted by the order statistics principle, the textures resist discrimination. However, as with the $U$ micropatterns, another explanation for the difficulty of discrimination is that, while appearing different in shape (micropattern X, Y, Z, and $P$ has a trapezoidal or $C$ shape, while micropattern $X, Y, Z$, and $Q$ has a $Y$ shape), the micropatterns are very much alike in a template matching sense; three of the four dots of the micropatterns would overlap exactly. In order to test this explanation, we created textures from the four micropatterns shown in Figure 5. They each contain four dots, three of which are positioned identically in all four micropatterns. In micropatterns $a$ and $b$, the fourth dots were positioned as described above, such that textures created from these two micropatterns would have identical second OS. For micropatterns $c$ and $d$, the midpoint of a different side of the triangle formed by the three common dots were selected, and the fourth dots were positioned such that the textures created from micropatterns $\mathrm{c}$ and $\mathrm{d}$ would agree in second OS. However, texture pairs consisting of one texture created from 


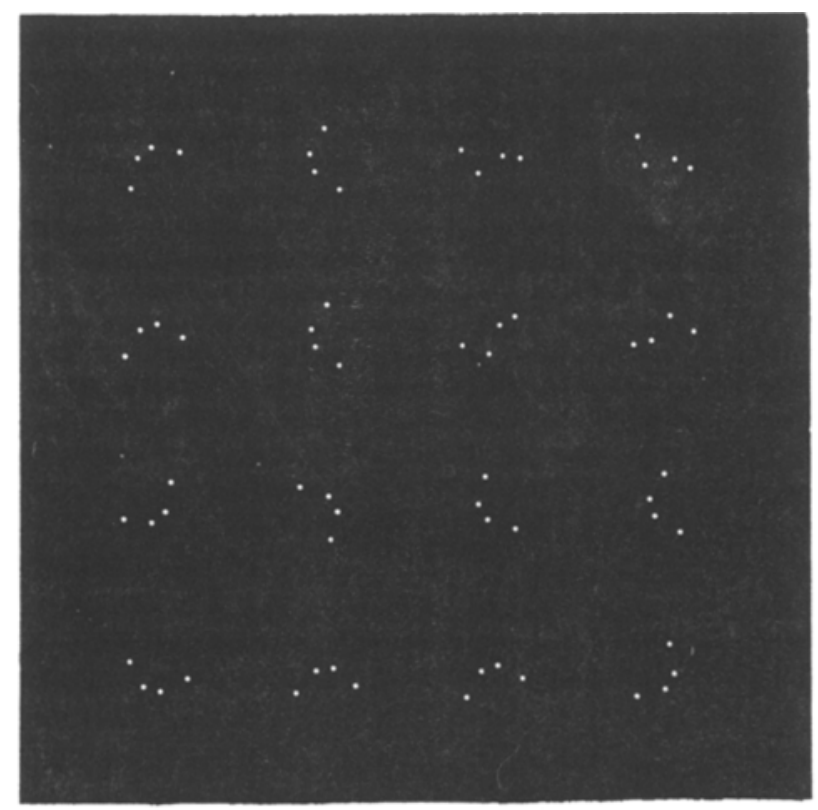

a.

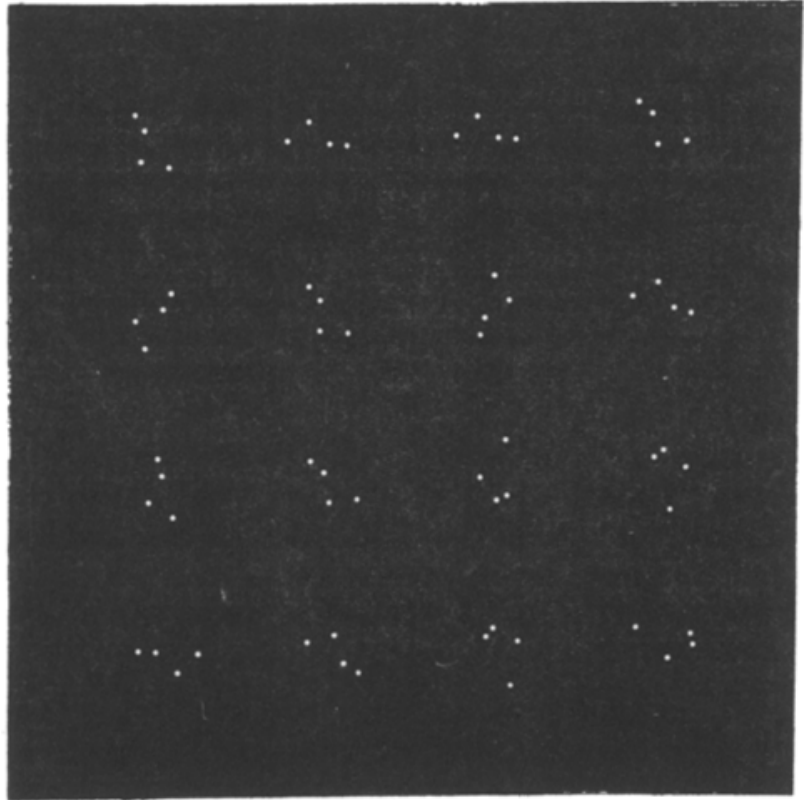

b.

Figure 6. Texture pair a has identical second-order statistics, while texture pair b has different second-order statistics. According to the order statistics principle, pair a should be difficult to discriminate and pair b easy to discriminate.

either micropattern a or micropattern $b$ and one texture created from either micropattern c or micropattern $d$ would have different OS. Thus, texture pairs created from a and $c, a$ and $d, b$ and $c$, and $b$ and $d$ would have different second $O S$.

\section{Method}

Subjects. A new group of 10 college students was used. Subjects were paid for their participation.
Stimuli and Procedure. The stimulus arrays were constructed as in the previous experiments with the 16 micropatterns in a square matrix that subtended a visual angle of $8^{\circ}$. The micropatterns used were those shown in Figure 5. The micropatterns were randomly rotated for reasons discussed above. All six possible texture pairs were presented. For each texture pair, there were eight different arrays. In four of these, one of the pair of textures filled the surround, and in the other four filled the disparate quadrant. For each surround-odd quadrant texture pair, there was one array for each of the possible disparate quadrants. There were thus 48 different arrays. Two of the texture pairs are shown in Figure 6. The procedure was the same as that used in Experiments 1 and 2, except that a block consisted of 48 trials.

\section{Results}

The RT data obtained are shown in Table 2. A one-way analysis of variance on the texture pairs showed a highly significant difference among the means for the six different texture pairs $[F(5,56)$ $=15.1, \mathrm{p}<.001]$. However, the differences between the means have little relationship to the statistical difference between texture pairs. For example, while both the a-b and c-d pairs agree in second OS, the $a-b$ discrimination was the most difficult of the six tested, while the c-d discrimination was the second easiest. A planned comparison of the weighted sum of the two texture pairs having second OS the same against the four pairs having second OS different was not significant $(F<1.00)$, and accounted for only $.4 \%$ of the variance. What did matter, unexpectedly, was whether the texture created from micropattern $d$ was one member of the pair of textures to be discriminated; when it was, RTs averaged $1,979 \mathrm{msec}$, whereas when it was not, they averaged $3,275 \mathrm{msec}$. This contrast was highly significant by a Scheffé post hoc comparison $(p<.001)$, and accounted for $97 \%$ of the variance among the means shown in Table 2.

\section{Discussion}

As in Experiments 1 and 2, when degree of overlap is controlled, no difference in discriminability was found between those texture pairs that differed in second OS and those that had the same second OS.

Table 2

Mean Reaction Times (in Milliseconds) for Texture Pairs Created from Dot Micropatterns

\begin{tabular}{|c|c|c|c|c|c|c|c|}
\hline \multicolumn{8}{|c|}{ Order Statistics } \\
\hline \multicolumn{4}{|c|}{ Second Order Same } & \multicolumn{4}{|c|}{ Second Order Different } \\
\hline $\begin{array}{l}\text { Pat- } \\
\text { tern }\end{array}$ & $\mathbf{R T}$ & $\begin{array}{l}\text { Error } \\
\text { Rate* }\end{array}$ & $\begin{array}{c}\text { Mean } \\
\text { RT }\end{array}$ & $\begin{array}{l}\text { Pat- } \\
\text { tern }\end{array}$ & RT & $\begin{array}{l}\text { Error } \\
\text { Rate* }^{*}\end{array}$ & $\begin{array}{c}\text { Mean } \\
\text { RT }\end{array}$ \\
\hline $\begin{array}{l}a-b \\
c-d\end{array}$ & $\begin{array}{l}3,400 \\
1,938\end{array}$ & $\begin{array}{l}4.0 \\
1.0\end{array}$ & 2,669 & $\begin{array}{l}a-c \\
a-d \\
b-c \\
b-d\end{array}$ & $\begin{array}{l}3,174 \\
1,830 \\
3,250 \\
2,180\end{array}$ & $\begin{array}{l}1.5 \\
1.5 \\
3.5 \\
1.5\end{array}$ & 2,606 \\
\hline
\end{tabular}

Note-Average for pairs including pattern $d=1,979$. Average for pairs not including pattern $d=3,275$.

*In percentages. 
However, RTs for the dot micropatterns were much longer than those found for the letter-like micropatterns. We therefore would not claim that we had found an example of textures which agree in second OS but which are immediately discriminable and do not require close scrutiny. Using the technique described above for creating dot micropatterns which agree in second OS, it is necessary to have the micropatterns overlap on three out of four dots. Because of this high degree of overlap, one would expect to find long RTs for discrimination. The significance of the finding of Experiment 3 is that one can create textures from micropatterns which differ in second-order statistics but, because of the high degree of overlap, are very difficult to discriminate.

The significant effect for pattern d could not be predicted on the basis of the order statistics principle, since textures created from d were paired both with textures that agreed in second OS and with textures that differed in second OS. There appears to be something distinctive about pattern $d$ itself that makes textures created from it easier to discriminate from other textures. Perhaps pattern $d$ forms more of a "good pattern" than the other three micropatterns, since it is more nearly symmetric (about a vertical axis as positioned in Figure 5). According to Garner (1974), good patterns are more easily encoded, and thus result in faster discriminations.

\section{EXPERIMENT 4}

In view of our three successive failures to confirm the order statistics principle, we considered the possibility that the arrays we had used were not perceived by our subjects as textures at all but rather as a collection of 16 discrete micropatterns. Julesz has hypothesized that the visual analyzers which detect texture differences operate only across small distances (both within and between micropatterns). Thus, if the micropatterns were too large, it is possible that mechanisms for form recognition rather than texture discrimination would be involved (Julesz, 1975). In another context (that of word recognition), Purcell, Stanovich, and Spector (1978) have demonstrated how the size of a visual array can have an important effect on the size of the visual units that are processed. While the $8^{\circ}$ arrays we used should have been sufficiently small to insure texture perception, given that Julesz' $(1973,1975)$ demonstrations were about this size, the experiments were repeated using arrays consisting of 64 micropatterns and subtending a visual angle of $4^{\circ} 36^{\prime}$. The size of the individual micropatterns relative to the size of the spaces between them was the same as in the previous experiments.

\section{Method}

Subjects. Six college students with normal vision served as subjects and were paid for their participation.

Stimuli. The stimulus arrays contained 64 micropatterns arranged in a square matrix. Of the 64 micropatterns, 48 were identical and filled the surround, while the disparate quadrant comained 16 identical micropatterns different from those in the surround. The 16 arrays from Experiment 1 using the $R$ micropatterns, the 16 arrays from Experiment 2 using the $\mathrm{L}$ micropatterns, and the 48 arrays from Experiment 3 using the dot micropatterns werereconstructed in a manner analogous to that described previously, only using 64 micropatterns instead of 16 per array.

Procedure. The arrays were presented on a computer-controlled cathode ray tube as in the previous experiments. The subjects were required to make the same buttonpressing response to indicate which quadrant contained the disparate array.

Stimuli were presented in blocks of 16 arrays each for the $\mathbf{R}$ and $L$ micropatterns and blocks of 48 arrays for the dot micropatterns. The subjects saw four trial blocks of the $\mathbf{R}$ micropatterns, four of the $\mathrm{L}$ micropatterns, and four of the dot micropatterns. Half of the subjects saw the dot micropatterns first followed by the $R$ and $L$ blocks, while the other half saw the $R$ and $L$ micropattern blocks first followed by the dot micropatterns.

\section{Results}

The results of this experiment are shown in Table 3. The $R$ and $L$ data were analyzed separately from those obtained for the dot micropatterns. A threeway analysis of variance of Patterns ( $R$ or $L$ ) by Order Statistics (second-order same or second-order different) by Reversals showed a significant main effect for order statistics only $[F(1,5)=10.9$, $\mathrm{p}<.05]$. No interactions were significant. As can be seen in Table 3 , for both the $R$ and $L$ micropatterns, texture pairs with second-order statistics the same were discriminated faster (mean $\mathrm{RT}=1,464$ ) than were the texture pairs with second-order statistics different (mean $\mathrm{RT}=1,706$ ), the reverse of the outcome predicted by the order statistics principle.

For the dot micropatterns, a one-way analysis of variance was performed on the six texture pairs. Differences among the means were highly significant $[F(5,25)=11.5, p<.001]$. As in Experiment 3, whether or not pattern $d$ was one member of the pair had a significant effect on discriminability. This comparison of texture pairs, including d vs. those not including $d$, was highly significant $[F(1,25)=$ $33.5, \mathrm{p}<.001]$ and accounted for $69 \%$ of the

Table 3

Mean Reaction Times (in Milliseconds) for Textures Consisting of 64 Micropatterns

\begin{tabular}{|c|c|c|c|c|c|c|}
\hline & \multicolumn{6}{|c|}{ Order Statistics } \\
\hline & \multicolumn{3}{|c|}{ Second Order Same } & \multicolumn{3}{|c|}{ Second Order Different } \\
\hline & $\begin{array}{l}\text { Pat- } \\
\text { tern }\end{array}$ & RT & $\begin{array}{c}\text { Mean } \\
\text { RT }\end{array}$ & $\begin{array}{l}\text { Pat- } \\
\text { tern }\end{array}$ & RT & $\begin{array}{c}\text { Mean } \\
\text { RT }\end{array}$ \\
\hline Letters & $\underset{\mathrm{L}}{\mathrm{R}} \stackrel{\mathrm{d}}{\mathrm{I}}$ & $\begin{array}{l}1,535 \\
1,392\end{array}$ & 1,464 & $\begin{array}{l}R \text { G } \\
\mathrm{L} I\end{array}$ & $\begin{array}{l}1,819 \\
1,593\end{array}$ & 1,706 \\
\hline Dots & $\begin{array}{l}a-b \\
c-d\end{array}$ & $\begin{array}{l}5,472 \\
3,114\end{array}$ & 4,293 & $\begin{array}{l}a-c \\
a-d \\
b-c \\
c-d\end{array}$ & $\begin{array}{l}4,060 \\
2,450 \\
3,781 \\
2,785\end{array}$ & 3,269 \\
\hline
\end{tabular}


variance among the six pattern pairs. However, unlike in Experiment 3, the comparison of texture pairs having second-order statistics the same vs. different was significant $[F(1,15)=13.45, p<.01]$ and in the direction predicted by the order statistics principle. In other words, it can be seen from Table 3 that the RTs for all three pairs containing a texture created from $d$ were shorter than for any of the texture pairs not including a texture created from d. However, within those pairs including $d$, the $c-d$ comparison was the longest; similarly for the three pairs not including $d$, the a-b comparison was the longest.

\section{Discussion}

In Experiment 4, an attempt was made to make the arrays appear more texture-like by reducing the size of the micropatterns and by including more micropatterns in each array. The results for the $\mathrm{R}$ and $\mathrm{L}$ micropatterns replicated those found in Experiments 1 and 2, with textures differing in second OS being more difficult to discriminate than those with the same second OS.

However, for the dot micropatterns, results in accord with the order statistics principle were obtained. Since this result was not obtained in Experiment 3, which used the same micropatterns at a larger size, this would indicate that, as Julesz has hypothesized, the visual analyzers which detect order statistics differences operate only across small distances.

Although the effect for order statistics was significant, it should be emphasized that other factors were affecting discriminability of the textures created from the dot micropatterns. In the first place, all of the RTs for the dot micropatterns were very long in this experiment compared to the preceding one. Although many factors not related to texture discriminability could elevate RTs (such as dimness of displays or difficulty of response), these long RTs may indicate that discrimination was not immediate for any of the texture pairs and that search was required in order to locate the discrepant texture. If this is correct, then we have demonstrated texture pairs which resist discrimination even when second-order statistics are different (namely texture pairs created from $a$ and $c$ and from $b$ and $c$ ). The significant effect for pattern $d$ also cannot be explained on the basis of order statistics. Perhaps when scrutiny is involved, a Gestalt property like goodness of pattern can affect discriminability. Goodness of pattern (or symmetry) cannot be predicted on the basis of second-order statistics, since, while patterns $c$ and $d$ have the same second-order statistics, pattern $d$ is nearly symmetric and pattern $c$ is not.

\section{GENERAL DISCUSSION}

Julesz' order statistics principle states that no two textures can be perceptually discriminated if they have identical second-order statistics. The results from the experiments using the $R$ and $L$ micropatterns provide counterevidence to this principle. ${ }^{4}$ While Experiment 4 provided some positive evidence for the difficulty in discrimination of patterns agreeing in second OS, it is also clear that this is not the only factor necessary to treate textures which resist discrimination. Although the demonstrations of textures consisting of dot micropatterns (Julesz, 1973, 1975) certainly do resist discrimination, a high degree of spatial overlap (three out of four dots in common) can also result in difficult discrimination, even for patterns that differ in second OS.

Evidence from other research also indicates that texture discriminability cannot be predicted solely on the basis of statistical properties. Olson and Attneave (1970), who examined a large number of textures, found that the ease with which a disparate texture could be located depended critically on the orientation of the entire array. ${ }^{5}$ Since rotating an array containing two textures does not alter the statistical relationship between the textures, it is clear that the order statistics principle cannot account for their large differences in discriminability. Related to this finding is the previously mentioned evidence concerning asymmetries in texture discriminability: a given pattern $\mathbf{A}$ is often more discriminable when placed against a background of $B$ than when $B$ is placed against a background of A (Frith, 1974; Reicher et al., 1976), although the statistical differences between A and B would, of course, remain the same. We obtained such asymmetries in Experiments 1 and 2 with the $R$ and $L$ micropatterns.

We conclude that texture discrimination cannot be fully explained on the basis of Julesz' order statistics principle. Several other factors affecting texture discrimination are suggested by the present results. The first of these is the previously discussed factor of similarity of micropatterns as measured by spatial overlap, which has been shown to be relevant to the discrimination of both letter and dot micropatterns.

However, this principle alone could not explain all of our findings. If positioned to obtain maximum overlap, the $\mathbf{R}$ and mirror-image $\mathrm{R}$ (as well as the $\mathrm{L}$ and mirror-image $L$ ) would overlap to the same extent as the $180^{\circ}$ rotations; however, the mirror images were considerably more difficult to discriminate than the rotations. This raises a second possible factor of border differences (Rosenfeld, 1967): perhaps the textures containing the $180^{\circ}$ rotations were easier to discriminate because of 
greater discontinuities at the border between the two textures. In Julesz' example with the U micropattern, disks were drawn around the individual micropatterns. Surrounding the $R$ and $L$ micropatterns with disks would serve to reduce possible border discontinuities. However, the disks would also increase spatial overlap of the micropatterns, and introduce extraneous factors, such as masking effects. Moreover, according to the order statistics principle, disks are not necessary to create micropatterns having the same second OS. Their use could only obscure the presence of border discontinuities, a factor which could not be predicted from the statistical differences between the two textures.

In order to handle border differences, the order statistics hypothesis would have to be modified so that order statistics were computed not just within micropatterns but across micropatterns. Julesz, as mentioned above, has hypothesized that texture analyzers operate only across small distances, and Gilbert and Shepp (Note 1), in their quantitative analysis of order statistics, have focused only on cases where the distances between micropatterns exceeds the distances within micropatterns (as in the case with all of our present stimuli). In arrays where Julesz claims no differences exist in second-order statistics (such as the $U$ vs. inverted- $U$ array), there do in fact exist perturbations in second-order statistics at the boundary between the two textures, but Julesz has assumed that these statistical differences are defined over such great relative distances that they do not affect the texture discrimination process. In order to explain our results from, say, Figure 1 within an order statistics framework, one would have to show that the perturbations in secondorder statistics that occur at the border are greater in $1 \mathrm{a}$ than in $1 \mathrm{~b}$, in fact, so much greater that they more than outweigh the greater statistical differences within micropatterns in $1 \mathrm{~b}$ than in 1a. It is not clear how to measure these border differences across textures, much less to compare their magnitudes to statistical differences within textures. In any case, the order statistics hypothesis would require major modifications to handle border differences successfully; as the hypothesis now stands, it cannot deal with them at all.

Thus, while we did find evidence for the effect of second-order statistics in Experiment 4 using randomly rotated dot micropatterns, several other factors are important in predicting texture discriminability. Although it is possible that all these diverse factors-spatial overlap, border differences, and order statistics-may yet be subsumed under a single, yet undiscovered principle, for the moment at least several principles seem necessary to explain texture perception.

\section{REFERENCE NOTE}

1. Gilbert. E. N.. \& Shepp, L. A. Textures for discrimination experiments. Bell Laboratories Technical Report, 1974.

\section{REFERENCES}

FRITH, U. A curious effect with reversed letters explained by a theory of schema. Perception \& Psychophysics, 1974, 16, 113-116.

GARNER, W. R. The processing of information and structure. Potomac, Md: Erlbaum, 1974.

Gibson, J. J. The perception of the visual world. Boston, Ma: Houghton-Mifflin, 1950.

Julesz, B. Visual pattern discrimination. IRE Transactions on Information Theory, 1962, IT-3, 84-92.

Julesz, B. Experiments in the visual perception of texture. Scientific American, 1975, 232, 34-43.

Julesz, B., Gilbert, E. N., Shepp, L. A., \& Frisch, H. L. Inability of humans to discriminate between visual textures that agree in second order statistics-revisited. Perception. 1973, 2, 391-405.

Olson, R., \& Attneave, F. What variables produce similarity grouping? American Journal of Psychology, 1970, 83, 1-21.

Pomerantz, J. R., Sager, L. C., \& Stoever, R. J. Perception of wholes and their component parts: Some configural superiority effects. Journal of Experimental Psychology: Human Perception and Performance, 1977, 3, 422-435.

Purcell, D. G., Stanovich, K. E., \& Spector, A. Visual angle and the word superiority effect. Memory \& Cognition, 1978, 6, 3-8.

Reicher, G., SNyder, C., \& Richards, J. Familiarity of background characters in visual scanning. Journal of Experimental Psychology: Human Perception \& Performance, 1976, 2, $522-530$.

ROSENFELD, A. On models for the perception of visual texture. In W. Wathen-Dunn (Ed.), Models for the perception of speech and visual form. Cambridge, Mass: MIT Press, 1967.

\section{NOTES}

1. However, the reverse cannot be true. If two textures differ in first-order statistics (i.e., one has a higher density of black dots), then it necessarily follows that, for at least one pin of a particular length and orientation, the probability of both ends landing on black will be greater for the texture with higher density. In general, if two textures differ in the $i^{\text {th }}$ order statistics, they will differ in $\mathrm{j}^{\text {th }}$ order statistics for all $\mathrm{j}>\mathrm{i}$.

2. Julesz does not specify a particular viewing distance for his demonstrations, nor, as he claims, do his effects seem to vary with viewing distance over a wide range. In Julesz (1975), the arrays printed would subtend visual angles of approximately $11.4^{\circ}$ and $5.7^{\circ}$ when viewed from distances of 25.4 and $50.8 \mathrm{~cm}$, respectively.

3. In informal tests using JuIsez' arrays, which he claims produce "spontaneous" texture discrimination (such as his U vs. C discrimination), we have found that RTs to locate the disparate texture have exceeded $10 \mathrm{sec!}$ This indicates that even Julesz' own arrays do not provide strong support for his hypothesis that second-order statistical differences yield immediate, spontaneous discrimination.

4. Since completing this research, we have learned from Julesz (personal communication) that he has discovered some further counterexamples to the order statistics hypothesis, some of which appear to be different from ours. On the basis of these counterexamples, Julesz has now substantially reformulated the order statistics hypothesis.

5. For example, if arrays $3 \mathrm{a}$ and $3 \mathrm{~b}$ are each rotated $45^{\circ}$ clockwise, discriminating the disparate texture will become easier in $3 b$ relative to $3 \mathrm{a}$. 